\title{
Heterogeneous Dynamics of Coarsening Systems
}

\author{
P. Mayer, ${ }^{1}$ H. Bissig, ${ }^{2}$ L. Berthier,${ }^{3,4}$ L. Cipelletti,${ }^{5}$ J.P. Garrahan,${ }^{6}$ P. Sollich, ${ }^{1}$ and V. Trappe ${ }^{2}$ \\ ${ }^{1}$ Department of Mathematics, King's College, Strand, London, WC2R 2LS, UK \\ ${ }^{2}$ Physics Department, Université de Fribourg, 1700 Fribourg, Switzerland \\ ${ }^{3}$ Rudolf Peierls Centre for Theoretical Physics, University of Oxford, 1 Keble Road, Oxford, OX1 3NP, UK \\ ${ }^{4}$ Laboratoire des Verres UMR 5587, Université Montpellier II and CNRS, 34095 Montpellier, France \\ ${ }^{5}$ GDPC UMR 5581, Université Montpellier II and CNRS, 34095 Montpellier, France \\ ${ }^{6}$ School of Physics and Astronomy, University of Nottingham, Nottingham, NG7 2RD, UK
}

(Dated: June 19, 2018)

\begin{abstract}
We show by means of experiments, theory and simulations, that the slow dynamics of coarsening systems displays dynamic heterogeneity similar to that observed in glass-forming systems. We measure dynamic heterogeneity via novel multi-point functions which quantify the emergence of dynamic, as opposed to static, correlations of fluctuations. Experiments are performed on a coarsening foam using Time Resolved Correlation, a recently introduced light scattering method. Theoretically we study the Ising model, and present exact results in one dimension, and numerical results in two dimensions. For all systems the same dynamic scaling of fluctuations with domain size is observed.
\end{abstract}

Glassy and jammed materials display similar phenomenology, characterized in particular by slow and nonequilibrium dynamics, whose microscopic origin is still being actively investigated [1, 2, 3]. Recent research has shown that despite the absence of static ordering, glass-formers exhibit non-trivial spatial correlations of the local dynamics, resulting in dynamic heterogeneity [4, 5]. Here, we take the view that slow dynamics is intrinsically associated to dynamic heterogeneity, as suggested by studies of the glass transition $[6]$.

Dynamic heterogeneity has to be measured by means of statistical correlators that probe more than two points in space and time. An ideal experiment or calculation would compare local configurations around position $r$ at times $t$ and $t+\Delta t$ via a two-time quantity, $F(r, t, \Delta t)$. Traditionally, only the dynamics averaged over $t, r$, or thermal histories is discussed, $F_{\mathrm{av}}(\Delta t)=\left\langle\frac{1}{V} \int_{V} d^{d} r F(r, t, \Delta t)\right\rangle$. In a glass-former, $F_{\text {av }}$ could be for example the selfintermediate scattering function at a given wavevector. By contrast, our goal is to detect spatial correlations of the local dynamics. A natural correlator is [4, 5, , 6

$C(r, \Delta t)=\frac{1}{V} \int_{V} d^{d} r^{\prime}\left\langle F\left(r^{\prime}, t, \Delta t\right) F\left(r^{\prime}+r, t, \Delta t\right)\right\rangle-F_{\text {av }}^{2}(\Delta t)$,

built from two-point, two-time quantities. It is easier to measure the volume integral of (1), a dynamic susceptibility $\chi(\Delta t) \equiv \int_{V} d^{d} r C(r, \Delta t)$, which can be rewritten as the variance of the fluctuations of the two-time dynamics,

$$
\chi(\Delta t)=V\left[\left\langle\left(\frac{1}{V} \int_{V} d^{d} r F(r, t, \Delta t)\right)^{2}\right\rangle-F_{\mathrm{av}}^{2}(\Delta t)\right] .
$$

Physically, dynamic fluctuations increase when the number of independent dynamic objects decreases, but normalizations ensure that $\chi$ remains finite in the thermodynamic limit, except at a dynamic critical point, as discussed for supercooled liquids [7].
In this paper, we focus on coarsening phenomena, as one of the simplest and better understood physical situations characterized by slow dynamics and aging [8]. We introduce new experimental and theoretical methods to access $\chi$ and $C$, and show that coarsening systems display heterogeneous dynamics similar to that observed at equilibrium in glass formers. We perform experiments on a coarsening soft material, a dry foam, and we study the
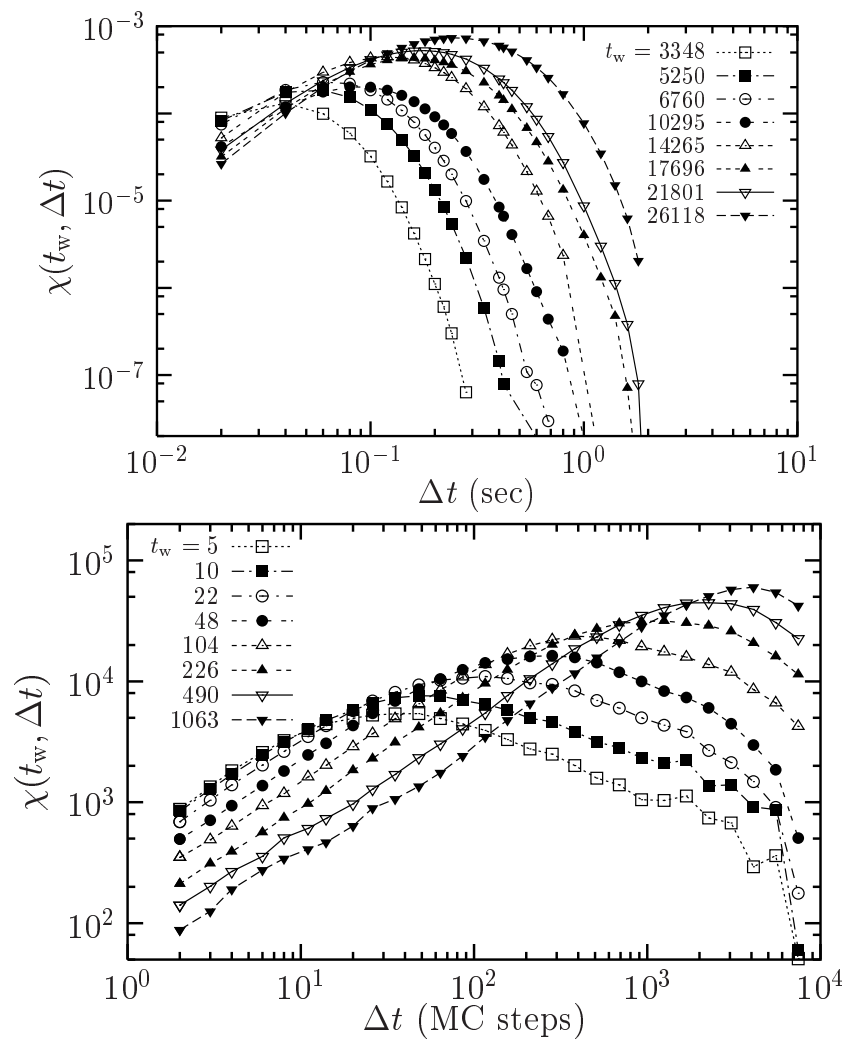

FIG. 1: Dynamic susceptibility $\chi\left(t_{\mathrm{w}}, \Delta t\right)$ measured experimentally in a coarsening foam (top), and numerically in the $d=2$ Ising model (bottom) for various ages $t_{\mathrm{w}}$. 
Ising model in one and two dimensions. Although very different, these systems display the same dynamic scaling of fluctuations with domain size.

Experimentally, soft materials are well suited for investigating dynamic heterogeneity because the relevant time and length scales are much larger than in molecular systems, which greatly simplifies detailed dynamic measurements. We use dynamic light scattering in the strongly multiple scattering limit (Diffusing Wave Spectroscopy, DWS 9]) to probe the dynamics of a shaving cream. The foam coarsens as the time $t_{\mathrm{w}}$ since its preparation increases. The time-averaged intensity autocorrelation function measured by DWS, $g_{2}\left(t_{\mathrm{w}}, \Delta t\right)-1$, decays exponentially, with a characteristic rate, $\Gamma\left(t_{\mathrm{w}}\right)$, that decreases with $t_{\mathrm{w}}$. This results from spatially and temporally localized random rearrangements 10].

Traditional light scattering measurements use a point detector and require an extended time average of the intensity correlation function. Consequently, no information on the fluctuations of the dynamics is accessible. To overcome this limitation, we use the recently introduced Time Resolved Correlation technique [11]. A charge-coupled device (CCD) camera is used to record, at a constant rate, the speckle pattern of the light scattered by the foam [12]. The degree of correlation, $c_{I}$, between speckles at times $t_{\mathrm{w}}$ and $t_{\mathrm{w}}+\Delta t$ is calculated as

$$
c_{I}\left(t_{\mathrm{w}}, \Delta t\right)=\frac{\left\langle I_{p}\left(t_{\mathrm{w}}\right) I_{p}\left(t_{\mathrm{w}}+\Delta t\right)\right\rangle_{p}}{\left\langle I_{p}\left(t_{\mathrm{w}}\right)\right\rangle_{p}\left\langle I_{p}\left(t_{\mathrm{w}}+\Delta t\right)\right\rangle_{p}}-1,
$$

where $I_{p}\left(t_{\mathrm{w}}\right)$ is the intensity measured at time $t_{\mathrm{w}}$ by the $p$-th CCD pixel and $\langle\cdots\rangle_{p}$ denotes an average over all pixels. Since different configurations yield different speckle patterns, $c_{I}\left(t_{\mathrm{w}}, \Delta t\right)$ quantifies the degree of correlation of the foam between times $t_{\mathrm{w}}$ and $t_{\mathrm{w}}+\Delta t$. Because the experiments are performed in the transmission geometry and in the strong multiple scattering regime, each CCD pixel collects light coming from the whole scattering volume. Therefore, $c_{I}\left(t_{\mathrm{w}}, \Delta t\right)$ provides time-resolved but spatially-integrated dynamical information. The standard intensity correlation function is obtained via a further average, $g_{2}\left(t_{\mathrm{w}}, \Delta t\right)-1=\left\langle c_{I}\left(t_{\mathrm{w}}, \Delta t\right)\right\rangle_{T}$, which is analogous to the correlator $F_{\mathrm{av}}$ defined above. Here, $\langle\cdots\rangle_{T}$ denotes an average over $\left[t_{\mathrm{w}}, t_{\mathrm{w}}+T\right]$, with $T \gg \Gamma^{-1}$, but short enough to prevent any significant change of the dynamics due to coarsening. In pratice, we took $T<0.05 t_{\mathrm{w}}$ for all ages, and checked that our results do not depend on this choice. Dynamic fluctuations are quantified via the variance of $c_{I}$, similarly to Eq. (2): $\chi\left(t_{\mathrm{w}}, \Delta t\right)=\left\langle c_{I}^{2}\left(t_{\mathrm{w}}, \Delta t\right)\right\rangle_{T}-\left\langle c_{I}\left(t_{\mathrm{w}}, \Delta t\right)\right\rangle_{T}^{2}$.

The dynamic susceptibility $\chi\left(t_{\mathrm{w}}, \Delta t\right)$ measured during the aging of the foam, from $t_{\mathrm{w}}=3350$ to $26200 \mathrm{sec}$ is shown in Fig. 1 Trivial contributions to the fluctuations due to the CCD noise and the finite number of speckles have been subtracted from the data 13. For all $t_{\mathrm{w}}$, we find that $\chi$ exhibits a peak at time lags $\Delta t^{\star}\left(t_{\mathrm{w}}\right)$ close to $\Gamma^{-1}\left(t_{\mathrm{w}}\right)$. Moreover, as the foam ages and coarsens, the height of the peak, $\chi^{\star}\left(t_{\mathrm{w}}\right)=\chi\left(t_{\mathrm{w}}, \Delta t^{\star}\right)$ increases and its position shifts to larger times, in striking analogy with numerical observations in supercooled liquids [4, 5]. To our knowledge, no experimental measurement of the dynamic susceptibility was so far reported.

A similar behavior can be observed in a very different coarsening system. We study the dynamics of the Ising model on a regular lattice quenched from a random state to the ferromagnetic phase. Domains of positive and negative magnetization develop and grow with $t_{\mathrm{w}}$, in analogy with the bubbles of the foam. The Hamiltonian is $H=-\sum_{\langle i, j\rangle} \sigma_{i} \sigma_{j}$, the sum being over nearest neighbor pairs. From (1), one would naively study $C_{0}(l-$ $\left.k, t_{\mathrm{w}}, \Delta t\right)=\left\langle\sigma_{k}(t) \sigma_{k}\left(t_{\mathrm{w}}\right) \sigma_{l}(t) \sigma_{l}\left(t_{\mathrm{w}}\right)\right\rangle-\left\langle\sigma_{k}(t) \sigma_{k}\left(t_{\mathrm{w}}\right)\right\rangle^{2}$, where $t=\Delta t+t_{\mathrm{w}}$. However, $C_{0}$ is trivially dominated by equal-time two-point correlations: consider for instance the large time limit, where $C_{0}\left(l-k, t_{\mathrm{w}}, \Delta t \rightarrow \infty\right) \rightarrow$ $\left\langle\sigma_{k}(t) \sigma_{l}(t)\right\rangle\left\langle\sigma_{k}\left(t_{\mathrm{w}}\right) \sigma_{l}\left(t_{\mathrm{w}}\right)\right\rangle$. The appropriate correlator to consider is instead [14]

$$
\begin{aligned}
C\left(l-k, t_{\mathrm{w}}, \Delta t\right)= & -\left\langle\sigma_{k}(t) \sigma_{k}\left(t_{\mathrm{w}}\right) \sigma_{l}(t) \sigma_{l}\left(t_{\mathrm{w}}\right)\right\rangle \\
& +\left\langle\sigma_{k}(t) \sigma_{k}\left(t_{\mathrm{w}}\right)\right\rangle\left\langle\sigma_{l}(t) \sigma_{l}\left(t_{\mathrm{w}}\right)\right\rangle \\
& +\left\langle\sigma_{k}(t) \sigma_{l}(t)\right\rangle\left\langle\sigma_{k}\left(t_{\mathrm{w}}\right) \sigma_{l}\left(t_{\mathrm{w}}\right)\right\rangle \\
& -\left\langle\sigma_{k}(t) \sigma_{l}\left(t_{\mathrm{w}}\right)\right\rangle\left\langle\sigma_{k}\left(t_{\mathrm{w}}\right) \sigma_{l}(t)\right\rangle,
\end{aligned}
$$

where the relative signs are consequences of the fermionic nature of fluctuations in the Ising model. The corresponding susceptibility is defined as in Eq. (2),

$$
\chi\left(t_{\mathrm{w}}, \Delta t\right)=\sum_{n} C\left(n, t_{\mathrm{w}}, \Delta t\right) .
$$

Note that (5) is not positive definite, but to ease the comparison with previous research, definitions (4) and (5) are chosen so that these quantities are eventually positive.

Dynamic fluctuations in the $d=2$ Ising model are measured in Monte Carlo (MC) simulations. The simulated system, $L$, must be large enough that the mean domain size, $R\left(t_{\mathrm{w}}\right)$, satisfies $R\left(t_{\mathrm{w}}\right) \ll L$ at all times, but small enough not to average out the fluctuations. We used $L=600$ and averaged the results over $2 \cdot 10^{3}$ independent initial conditions, the total simulated time being $10^{4} \mathrm{MC}$ steps. Due to computational limitations, we could only measure $\chi\left(t_{\mathrm{w}}, \Delta t\right)$ as the linear integral of (4) measured along the $x$ and $y$ axes. As seen in Fig. 1] the observed dynamical fluctuations are similar to those observed for the foam.

This similarity lies in the common physical mechanism responsible for the slow, heterogeneous dynamics: the domain growth driven by the reduction of interfacial energy. As the system coarsens, the size of the regions that undergo correlated rearrangements increases, so that the number of independent regions in the probed volume, $N\left(t_{\mathrm{w}}\right)$, decreases, which in turn increases the amplitude of the dynamic fluctuations. One would therefore expect that $\chi^{\star}\left(t_{\mathrm{w}}\right) \sim N^{-1}\left(t_{\mathrm{w}}\right) \sim R^{d}\left(t_{\mathrm{w}}\right)$. 
To substantiate this prediction, we first consider an analytically solvable case. We study the non-equilibrium dynamics of the $d=1$ Ising model. The spin chain evolves according to standard Glauber rules 15]. From general expressions for two-time, multispin correlation functions following a quench derived in [16], we compute exactly the quantities (4) and (5). Although straightforward in principle, actual calculations require technically involved algebraic manipulations, detailed in [14].

Consider first equilibrium dynamics which we expect to be dynamically homogeneous. Indeed, we find that the correlation (4) vanishes exactly, $C^{\mathrm{eq}}(n, \Delta t)=$ $\lim _{t_{\mathrm{w}} \rightarrow \infty} C\left(n, t_{\mathrm{w}}, \Delta t\right)=0$, for arbitrary distances $n$, times $\Delta t$, and temperatures $T>0$. This implies that two-time multispin correlations factorize into two-spin static correlations, and shows that the correlator (4) is well suited to revealing the existence of non-trivial, dynamic, out of equilibrium correlations.

The situation is more interesting in the coarsening regime. We focus on the scaling behavior for large times $\Delta t, t_{\mathrm{w}} \rightarrow \infty$ and distances $n \rightarrow \infty$, with scaling variables $\alpha=\Delta t / t_{\mathrm{w}}$ and $\eta=n / R\left(t_{\mathrm{w}}\right)$ fixed, where $R\left(t_{\mathrm{w}}\right) \sim \sqrt{t_{\mathrm{w}}}$. One finds scale invariance,

$$
C\left(n, t_{\mathrm{w}}, \Delta t\right) \sim f_{C}\left(\frac{\Delta t}{t_{\mathrm{w}}}, \frac{n}{R\left(t_{\mathrm{w}}\right)}\right),
$$

where the scaling function $f_{C}(\alpha, \eta)$ is given in [14]. From (6), the susceptibility (5) scales as

$$
\chi\left(t_{\mathrm{w}}, \Delta t\right) \sim R\left(t_{\mathrm{w}}\right) F_{C}\left(\frac{\Delta t}{t_{\mathrm{w}}}\right),
$$

with $F_{C}(\alpha)=\int \mathrm{d} \eta f_{C}(\alpha, \eta)$. Both scaling functions are displayed in Fig. 2 In particular, $\chi\left(t_{\mathrm{w}}, \Delta t\right)$ qualitatively resembles the results in Fig. [1 and Eq. (7) shows that the peak height scales as expected, $\chi^{\star}\left(t_{\mathrm{w}}\right) \sim R\left(t_{\mathrm{w}}\right)$.

To develop a more direct understanding of (6), we note that the dynamics of the Ising spin chain can be mapped to a diffusion-limited annihilation process, where one studies the dynamics of the walls separating domains of opposite magnetization rather than that of the spins themselves 17]. Trajectories of the walls are random walks that annihilate whenever they meet. In a spacetime diagram [6], the spins $\sigma_{k}\left(t_{\mathrm{w}}\right), \sigma_{l}\left(t_{\mathrm{w}}\right), \sigma_{k}(t)$ and $\sigma_{l}(t)$ occupy the corners of a rectangle of size $n=k-l$ by $\Delta t=t-t_{\mathrm{w}}$, and the spin products in (4) are determined by the parity of the number of random walks crossing the relevant edge. Labeling edges in the order left - right - top - bottom we denote, for example, an odd number of random walkers crossing the left and bottom edges as 1001. Because walls annihilate in pairs, the number of walkers crossing the rectangle is even, so that there are only 8 possible situations. In terms of the corresponding probabilities, (4) may be reexpressed as $C\left(n, t_{\mathrm{w}}, \Delta t\right)=8\left(p_{0101} p_{1010}+p_{0110} p_{1001}-p_{0000} p_{1111}-\right.$ $\left.p_{0011} p_{1100}\right)$. These probabilities can be evaluated to leading order via standard random walk arguments [14], when

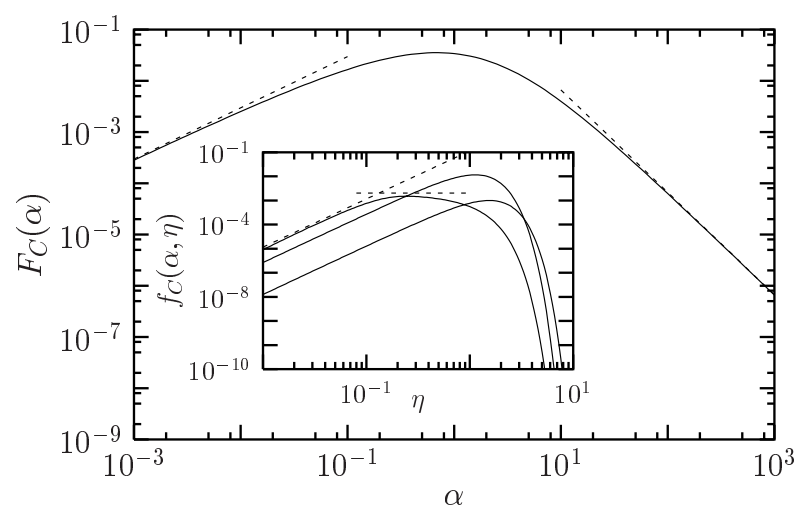

FIG. 2: Exact scaling function of Eq. (7) for the dynamic susceptibility of the Ising chain. Dashed lines show the asymptotic behavior, $F_{C}(\alpha \rightarrow 0) \sim \alpha$ and $F_{C}(\alpha \rightarrow \infty) \sim \alpha^{-2}$. Inset: Exact scaling function of Eq. (6), for $\alpha=10^{-2}, 1$ and 10 (from top to bottom on the left). Dashed lines represent the asymptotic behavior estimated via random walk arguments.

the number of trajectories crossing the rectangle is small, i.e. for diluted walls, $n \ll R\left(t_{\mathrm{w}}\right)$, and short time delays, $\Delta t \ll t_{\mathrm{w}}$. For $\Delta t \ll n^{2} \ll t_{\mathrm{w}}, C\left(n, t_{\mathrm{w}}, \Delta t\right)$ converges to an $n$-independent plateau of height $\left(2 / \pi^{2}\right) \alpha$, see Fig. 2] When $n^{2} \ll \Delta t \ll t_{\mathrm{w}}$, on the other hand, $C\left(n, t_{\mathrm{w}}, \Delta t\right) \approx \pi^{-1}(1-2 / \pi) n^{2} / t_{\mathrm{w}}$, implying that $C$ grows like $\eta^{2}$ with a $\Delta t$-independent amplitude, see Fig. 2 The random walk picture becomes too complicated when either $n^{2}$ or $\Delta t$ are large compared to $t_{\mathrm{w}}$ and we refer to our exact results in this regime [14]. For $n^{2} \ll t_{\mathrm{w}} \ll \Delta t$, the $\eta^{2}$-dependence found above for $n^{2} \ll \Delta t \ll t_{\mathrm{w}}$ persists, but now with an amplitude that decreases as $\alpha^{-2}$. For large $n^{2} \gg t_{\mathrm{w}}$, finally, we find a Gaussian cutoff in $n$ with a width of order $\sqrt{t_{\mathrm{w}}}$ for both $\Delta t \ll t_{\mathrm{w}}$ and $\Delta t \gg t_{\mathrm{w}}$.

The asymptotic behavior of $\chi\left(t_{\mathrm{w}}, \Delta t\right)$ follows from the above discussion. For $\Delta t \ll t_{\mathrm{w}}$, the integral is dominated by the plateau region of $f_{C}$. Since the plateau grows as $\alpha$, so does (7). For $\Delta t \gg t_{\mathrm{w}}$, on the other hand, the decrease in the amplitude of the $\eta^{2}$-part of $C$ controls the variation of $\chi$ which decays therefore as $\alpha^{-2}$. Rigorous analysis shows indeed $F_{C}(\alpha \rightarrow 0) \sim 4(\sqrt{2}-1) \pi^{-3 / 2} \alpha$ and $F_{C}(\alpha \rightarrow \infty) \sim(8 / 5)(8 \sqrt{2}-9) \pi^{-3 / 2} \alpha^{-2}$, see Fig. [2]

For the $d=2$ Ising model and the foam no analytical results are available. Instead, we test the scaling of $\chi\left(t_{\mathrm{w}}, \Delta t\right)$ by measuring $R\left(t_{\mathrm{w}}\right)$ and the characteristic relaxation time. In simulations, $R\left(t_{\mathrm{w}}\right)$ is determined from the decay at large distance of the equal-time structure factor, while the characteristic decay rate scales as $1 / t_{\mathrm{w}}$, $\Gamma\left(t_{\mathrm{w}}\right) \sim t_{\mathrm{w}}^{-1}[8]$. In the simulations, $\chi$ is a linear integral over distance, as mentioned above. Thus, one expects $\chi^{\star}\left(t_{\mathrm{w}}\right) \sim R\left(t_{\mathrm{w}}\right)$. For the foam, $R\left(t_{\mathrm{w}}\right)$ scales as the bubble size, which is also proportional to the photon transport mean free path, $\ell^{\star}\left(t_{\mathrm{w}}\right)$ [10], which we obtain independently from transmission measurements 9]. We expect therefore $\chi^{\star}\left(t_{\mathrm{w}}\right) \sim \ell^{\star 3}\left(t_{\mathrm{w}}\right)$.

As seen in Fig. 3. not only does the peak of the dynamic 

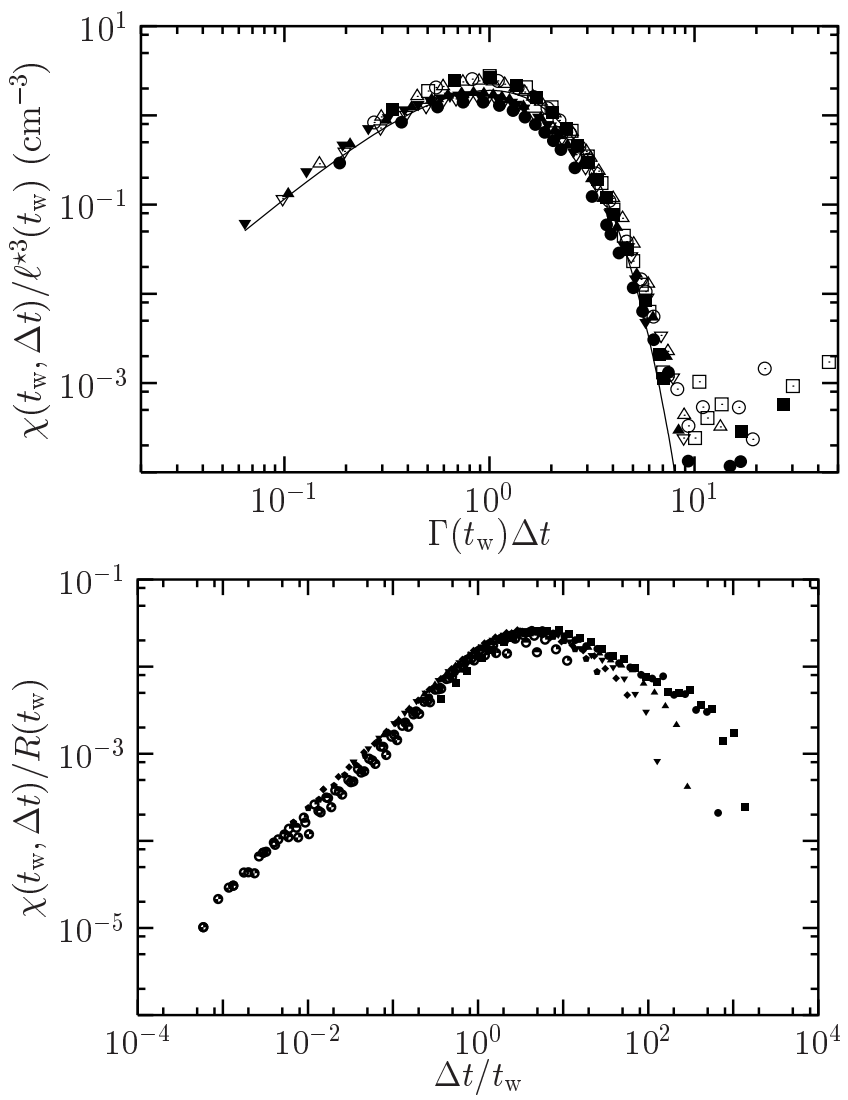

FIG. 3: Dynamic scaling of all dynamic susceptibilities in Fig. 1 (same symbols). Top: experiments on a foam, the line is the scaling function of Eq. (8). Bottom: numerical simulations of the $d=2$ Ising model.

susceptibility scale as expected, but data for all times collapse onto a master curve, a scaling behavior analogous to that found for the Ising chain, Eq. (7). For the foam, the shape of the master curve can be explained by noting that at all times, $c_{I}\left(t_{\mathrm{w}}, \Delta t\right) \sim \exp \left[-\gamma\left(t_{\mathrm{w}}\right) \Delta t\right]$, where $\gamma\left(t_{\mathrm{w}}\right)$ is a decay rate fluctuating with the number of rearrangements, of mean $\Gamma\left(t_{\mathrm{w}}\right)=\left\langle\gamma\left(t_{\mathrm{w}}\right)\right\rangle_{T}$. To leading order in the variance of $\gamma\left(t_{\mathrm{w}}\right), \sigma^{2}\left(t_{\mathrm{w}}\right) \equiv\left\langle\gamma\left(t_{\mathrm{w}}\right)^{2}\right\rangle_{T}-\left\langle\gamma\left(t_{\mathrm{w}}\right)\right\rangle_{T}^{2} \sim$ $\ell^{* 3}\left(t_{\mathrm{w}}\right) \Gamma^{2}\left(t_{\mathrm{w}}\right)$, one can estimate

$$
\chi\left(t_{\mathrm{w}}, \Delta t\right) \sim \ell^{* 3}\left(t_{\mathrm{w}}\right)\left[\Gamma\left(t_{\mathrm{w}}\right) \Delta t\right]^{2} e^{-2 \Gamma\left(t_{\mathrm{w}}\right) \Delta t} .
$$

The solid line in the top panel of Fig. 3 shows that the scaling function $f(x)=x^{2} \exp (-2 x)$, suggested by (8), is in very good agreement with the experimental data.

In summary, we have defined and analyzed dynamic spatial correlators and susceptibilities revealing the growth with time of dynamic heterogeneity in coarsening systems. The increasing dynamical fluctuations are consequences of a reduced number of independent dynamical domains - bubbles or magnetized domainsas coarsening proceeds. The non-fractal morphology of the domains implies a particularly simple scaling of spatial correlators, and therefore of their volume integral, $\chi\left(t_{\mathrm{w}}, \Delta t\right) \sim R^{d}\left(t_{\mathrm{w}}\right) f\left(\Gamma\left(t_{\mathrm{w}}\right) \Delta t\right)$. However, dynamic heterogeneity is also found in systems where dynamic domains are believed to have a fractal morphology [4, 5]. In this case, a more complicated dynamic scaling can be expected [4, 7]. The techniques presented in this work will help identify and characterize dynamic heterogeneity, an endeavor that appears as a crucial step in gaining a better understanding of the dynamic slowing down of many glassy and jammed materials.

We thank P. Ballesta and A. Lefèvre for useful discussions. We acknowledge financial and numerical support from EPSRC grants 00800822, GR/R83712/01 and GR/S54074/01, E.U. grant No. HPMF-CT-2002-01927, SNF Grant 2100-066920, CNRS (PICS 2410), French Ministère de la Recherche (ACI Jeunes Chercheurs), ESF Program SPHINX, Nuffield Grant NAL/00361/G, Österreichische Akademie der Wissenschaften, Worcester College Oxford, and Oxford Supercomputing Center.

[1] Slow relaxations and nonequilibrium dynamics in condensed matter, Eds: J.-L. Barrat, J. Dalibard, M. Feigelman and J. Kurchan (Springer, Berlin, 2003).

[2] P.G. Debenedetti and F.H. Stillinger, Nature (London) 410, 259 (2001).

[3] A.J. Liu and S.R. Nagel, Nature 396, 21 (1998).

[4] See e.g. E.R. Weeks, J.C. Crocker, A.C. Levitt, A. Schofield, and D.A. Weitz, Science 287, 627 (2000); W.K. Kegel and A. van Blaaderen, Science 287, 290 (2000); D. Perera and P. Harrowell, Phys. Rev. E 51, 314 (1995); C. Donati, S.C. Glotzer, P.H. Poole, W. Kob, and S.J. Plimpton, Phys. Rev. E 60, 3107 (1999); G. Parisi, J. Phys. Chem. B 103, 4128 (1999).

[5] For reviews see H. Sillescu, J. Non-Cryst. Solids 243, 81 (1999); M.D. Ediger, Annu. Rev. Phys. Chem. 51, 99 (2000); S.C. Glotzer, J. Non-Cryst. Solids 274, 342 (2000).

[6] J.P. Garrahan and D. Chandler, Phys. Rev. Lett. 89, 035704 (2002); L. Berthier and J.P. Garrahan, Phys. Rev. E 68, 041201 (2003).

[7] S. Whitelam, L. Berthier, and J.P. Garrahan, Phys. Rev. Lett. 92, 185705 (2004).

[8] A.J. Bray, Adv. Phys. 43, 357 (1994).

[9] D.A. Weitz and D.J. Pine, in Dynamic Light Scattering, Ed: W. Brown (Clarendon Press, Oxford, 1993).

[10] D.J. Durian, D.A. Weitz, and D.J. Pine, Science 252, 686 (1991).

[11] L. Cipelletti, H. Bissig, V. Trappe, P. Ballesta, and S. Mazoyer, J. Phys.: Condens. Matter 15, S257 (2003).

[12] J.W. Goodman, in Laser Speckles and Related Phenomena, Ed.: J.C. Dainty (Springer, Berlin, 1975).

[13] A. Duri, H. Bissig, V. Trappe, P. Ballesta, and L. Cipelletti, to appear in the proceedings of the SPIE conference.

[14] P. Mayer, L. Berthier, J.P. Garrahan, and P. Sollich, in preparation.

[15] R.J. Glauber, J. Math. Phys. 4, 294 (1963).

[16] P. Mayer and P. Sollich, J. Phys. A: Math. Gen. 37, 9 (2004).

[17] J.E. Santos, J. Phys. A: Math. Gen. 30, 3249 (1997). 